\title{
SOME STORIES OF BRASIL-JAPAN COLLABORATION AND CHACALTAYA MOUNTAIN
}

\author{
S.L.C. BARROSO ${ }^{1, *}$, P.C. BEGGIO ${ }^{2, * *}$, L.F. GOMEZ G. ${ }^{3, * * *}$, E.J.T. MANGANOTE ${ }^{4, * * *}$, A. MARIANO ${ }^{3, \dagger}$, \\ M.J. MENON ${ }^{3,}$, and E.H. SHIBUYA ${ }^{3, \S}$ \\ ${ }^{1}$ Departamento de Ciências Exatas e Tecnológicas, Universidade Estadual do Sudoeste da Bahia, 45083-900, Vitória da Conquista, BA, \\ Brasil. \\ ${ }^{2}$ Laboratório de Ciências Matemáticas, Universidade Estadual do Norte Fluminense, Campos dos Goytacazes, RJ, Brasil. \\ ${ }^{3}$ Instituto de Física Gleb Wataghin, Universidade Estadual de Campinas, 13083-970 Campinas, SP, Brasil. \\ ${ }^{4}$ Faculdades de Campinas, FACAMP, 13083-898 Campinas, SP, Brasil.
}

\begin{abstract}
.
An international cooperation, named Brasil-Japan Collaboration of Chacaltaya Emulsion Chamber Experiment (B-J Collaboration) exposed $2.35 \times 10^{8} \mathrm{~m}^{2} . \mathrm{s}$, during the period $15^{\text {th }} \mathrm{Jun}, 1962$ till $10^{\text {th }}$ May, 1988. It corresponds to 22 'runs' on Cosmic Rays and in this figure are not included photosensitive material set in the lower part of the two-storied emulsion chamber. Although 3 more 'runs' occurred after 1988, they were not included either, because they were not yet properly measured and because they contain mixed Japanese and Russian films. Chacaltaya Mountain was firstly used in 1947 for 2 meson observations and $\pi-\mu$ decays. It was a wise choice not only for these observations but also for the B-J Collaboration and for the Alpaca Project, running at neighbouring sites. The main results observed by the B-J Collaboration are presented.
\end{abstract}

\section{Historical introduction}

A series of experiments were carried out at the Observatory of Mount Chacaltaya $\left(5,220 \mathrm{~m}=540 \mathrm{~g} / \mathrm{cm}^{2}\right.$ level, geographic coordinates $16^{0} 20^{\prime} 45^{\prime \prime}$ South and $68^{\circ} 07^{\prime} 31^{\prime \prime}$ West corresponding to geomagnetic coordinates $4^{0} 50^{\prime} 40^{\prime \prime}$ South and $0^{\circ} 50^{\prime} 20^{\prime \prime}$ East, respectively). The purpose of these experiments was to investigate high energy interactions of Cosmic Rays as proposed by Hideki Yukawa to Cesare Mansueto Giulio Lattes, following a draft by Yoichi Fujimoto and Masatoshi Koshiba.

One of the predictions of Multiple Particle Production phenomena was made by Gleb Wataghin [1] interpreting the data obtained by Georg Photzer [2] exposing GeigerMuller detectors flown in balloon flights, especially the maximum intensity known as the Photzer maximum.

Exposing Ilford G5 Emulsion Plates placed in a vertical position, as was usual at that time, on board a ballon flying at $27 \mathrm{~km}$ above sea level, J.J.Lord et al.[3] obtained empirical evidence of Multiple Meson Production. Another clear evidence for this phenomena in a single collision was seen in a high-pressure cloud chamber filled with hydrogen gas [4].

\footnotetext{
*e-mail: serlc@gmail.com

**e-mail: beggio@uenf.br

***e-mail: lfgomez@gmail.com

****e-mail: ejtmanganote@gmail.com

†e-mail: andersom.mariano@gmail.com

†e-mail: marciojmenon@gmail.com

$\S_{\text {e-mail: ehiros@hotmail.com }}$
}

Subsequent experiments, for instance the I.C.E.F. Collaboration [5], observed the same phenomena and also that the jet is more collimated the higher the energy of the interaction. This fact inspired J.Nishimura to hypothesize a 'similarity law' [6], nowadays known as 'Feynman scaling' and at the same time to adapt a device with photosensitive material arranged in a horizontal position inserted between lead plates. So, the Emulsion Chamber works as a detector of electromagnetic showers induced mainly by $\pi^{0}$, but this arrangement caused additional difficulties to analyse interactions, that is:

1) to determine the interaction point (vertex of the interaction) and

2) discriminate between electromagnetic showers induced by $\gamma$ and hadronic particles (identification of the showers).

To study the Multiple Particle Production phenomena, mainly Multiple Meson Production, the B-J Collaboration exposed 25 emulsion chambers to cosmic ray particles arriving at Mount Chacaltaya level. The emulsion chamber consists of multi-layered envelopes containing typically 2 or $3 \mathrm{X}$-ray films and 1 nuclear emulsion plate, all having an area of $40 \mathrm{~cm} \times 50 \mathrm{~cm}$ and thickness of $200 \mu \mathrm{m}$ and $1,550 \mu \mathrm{m}$, respectively [7]. The envelopes are inserted between lead plates and the last 11 chambers have a two storey structure as the main detector. 68,825 X-Ray films and 14,211 Emulsion Plates, in total were used.

A typical emulsion chamber is composed of an upper chamber above blocks of asphalt pitch or compacted plastic sheets, both located on an iron frame platform and, below those, a lower detector separated by an air gap. The 


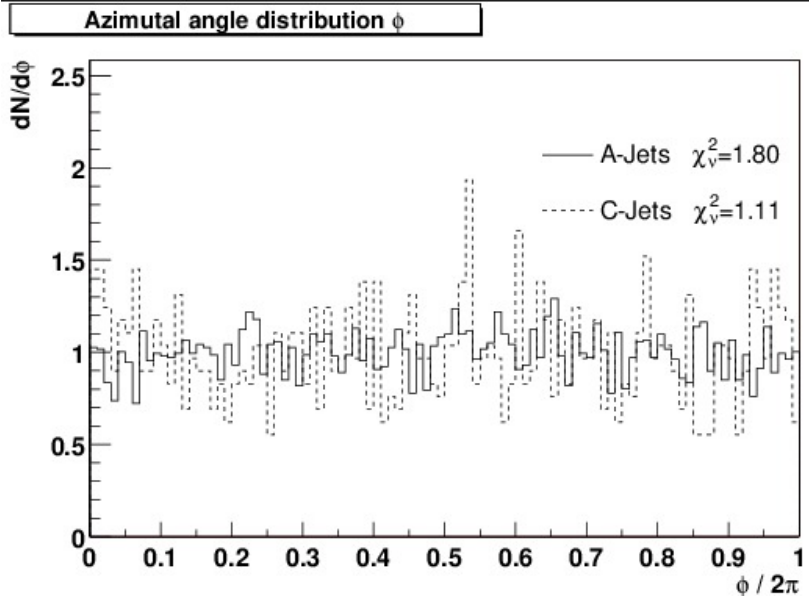

Figure 1. Azimuthal angle distribution.

analysis was made on a sample of 372 events, 87 of whioch were observed in only the lower detector. These events were called C-jets. The other 285 events, called A-jets, were observed in the upper detector and sometimes continuing to the lower part. Included in these A-jets sample were superposed interaction events (13 halo events) and 5 events, nicknamed Centauro events.

\section{Analysis Methods}

\subsection{Height (vertex) determination}

A method to determine the interaction point is to use the $\pi^{0}$ decay mode ( $98.8 \%$ of branching ratio) to $2 \gamma$-rays. In a very lucky case of the A-jets analysis (the event nicknamed Centauro V is the remarkable one) an increase of the geometrical distance between the $2 \gamma^{\prime} s$ from upper to lower chamber was observed. As C-jets originate in the Carbon target, located $170 \mathrm{~cm}$ above the lower chamber, their analysis is straightforward, choosing pairs of $\gamma^{\prime} s$ that determine the decay point of the $\pi^{0}$ inside the target.

\subsection{Algorithms R-r and mDW-r}

Beyond a traditional analysis done in some occasions, we suggested a method based on the azimuthal isotropic decay of secondaries observed in figure 1 . The superposed experimental distributions for A-jets and C-jets were compared with a uniform distribution and resulted in reduced $\chi^{2}$ values between 1.1 and 1.8 .

Based on this observed isotropic azimuthal angle distribution, the secondary particles distribution composed by energy $E^{*}$ and solid angle $\Omega^{*}$, expressed in a center of mass system is

$$
\left.d N=g\left(E^{*}, \Omega^{*}\right) d E^{*} d \Omega^{*}\right)
$$

transforms to a general expression for the moments below, where $\mathrm{E}_{i}$ and $\theta$ are the energy and zenith angle of the $\mathrm{i}^{\text {th }}$ particle, $n$ is the order of the moment, $\Gamma$ and $M$ are the Lorentz factor and Invariant Mass of the group of particles, respectively: $\mathrm{r} \times \mathrm{R}$

C-JET 15028007

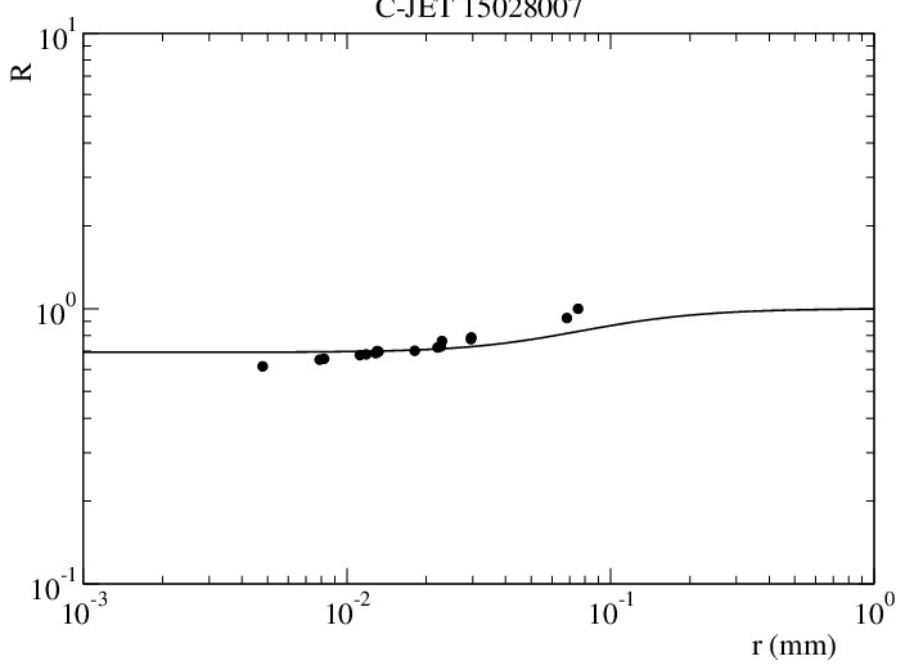

Figure 2. Example of algorithm $\mathrm{R}$ for $\mathrm{C}$-jet.

$$
\Sigma E_{i}\left(\Gamma \theta_{i}\right)^{n} \simeq M \Gamma \int_{\frac{1-\Gamma^{2} \theta^{2}}{1+\Gamma^{2} \theta^{2}}}^{1} \frac{\left(1-x^{2}\right)^{\frac{n}{2}}}{2(1+x)^{n-1}} d x
$$

with $\mathrm{x}=\cos \theta^{*}=\left[1-\left(\Gamma \theta_{i}\right)^{2}\right] /\left[1+\left(\Gamma \theta_{i}\right)^{2}\right]$. All the moments are functions of $\Gamma \theta$ and so, functions of $\mathrm{r} / \bar{r}$ either, because $\Gamma \theta=(\mathrm{H} / \bar{r}) .(\mathrm{r} / \mathrm{H})$, where $\mathrm{H} \approx$ interaction height. From the correlation R-r, $\bar{r}$ is obtainable for $\mathrm{R}=0.75$, because at this value $\Gamma \theta=1$. Therefore, the algorithms are useable even for events where the height is not determined.

Taking the $0^{\text {th }}, 1^{\text {st }}, 2^{\text {nd }}$ and $3^{\text {rd }}$ moments, a proper combination of them, each one normalized, algorithms called $\mathrm{R}$ and $\mathrm{mDW}$ were constructed. The first one, $\mathrm{R}$ it is defined by,

$$
R=\frac{\left[\Sigma E_{i}\right]\left[\Sigma E_{i}\left(\Gamma \theta_{i}\right)^{2}\right]}{\left[\frac{4}{\pi} \Sigma E_{i}\left(\Gamma \theta_{i}\right)\right]^{2}}
$$

where both the numerator and denominator are functions of $\Gamma \theta$ and the Squared Invariant Mass, as can be seen in Appendix A. So, the events that fit this analytical curve are events coming from one jet produced at one single interaction point. Examples of the application of algorithm $\mathrm{R}$ are presented in figures 2 and 3 for $\mathrm{C}$-jet and A-jet, respectively. This algorithm was first used in [8] to present a 'selective' production of $\eta$ mesons in A-jets. The same behaviour was seen in C-jets [9].

The second algorithm was called $\mathrm{mDW}$ due to its similarity to the Duller-Walker plot [10], used to show the 'sphericity' of a jet. It differs from that in the fact that it also uses the energy of each member of a jet and, a priori, does not need to know the total multiplicity. The events that fit this curve are interpretable as spherical because the slope is 2 , the same kind of reasoning for the isotropic characteristic coming from DW-plot. It is defined as,

$$
\begin{array}{r}
m D W=\frac{1}{4 M \Gamma}\left[\Sigma E_{i}+\frac{4}{\pi} \Sigma E_{i}\left(\Gamma \theta_{i}\right)+\Sigma E_{i}\left(\Gamma \theta_{i}\right)^{2}+\right. \\
\left.\frac{4}{3 \pi} \Sigma E_{i}\left(\Gamma \theta_{i}\right)^{3}\right]
\end{array}
$$




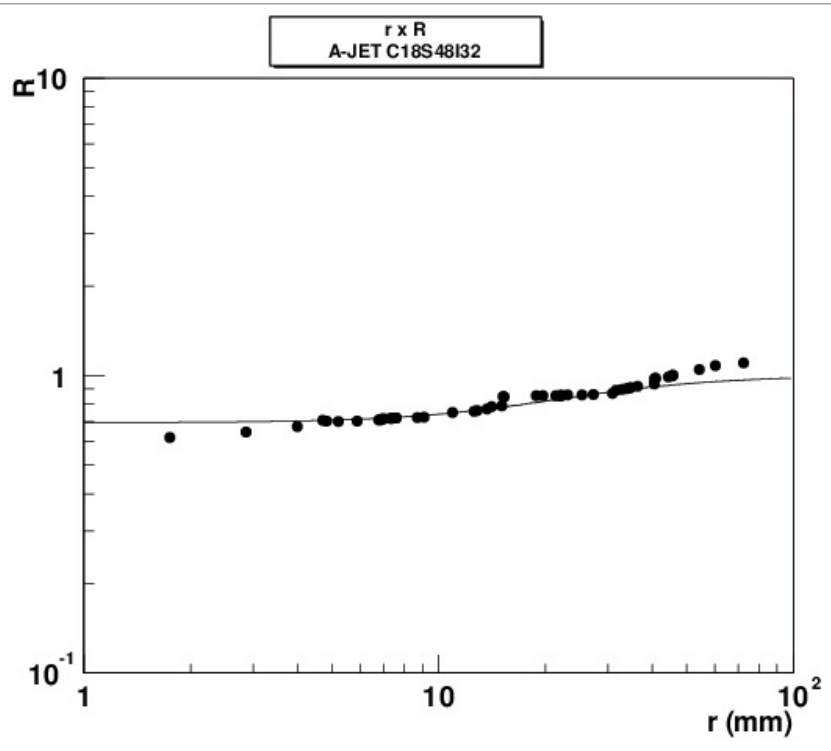

Figure 3. Example of algorithm R for A-jet.

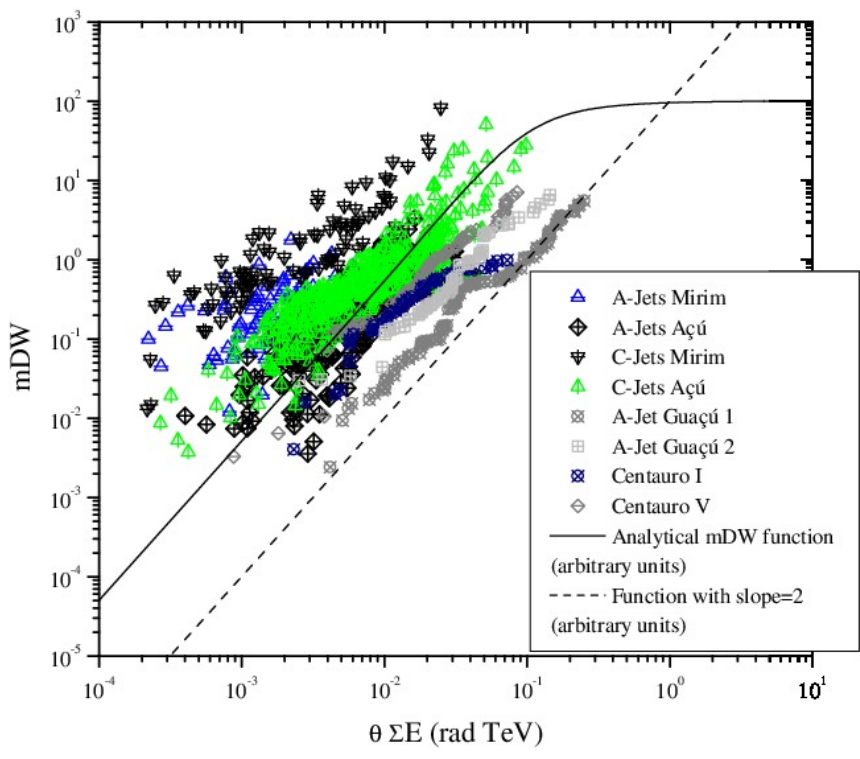

Figure 4. Algorithm mDW for some events with measured interaction point.

This algorithm was applied in figure 4 for various Ajets, C-jets and also for Centauro events with the height determined through the kinematics of $\pi^{0} \rightarrow \gamma+\gamma$ and the triangulation method. Defining 'sphericity' as the slope of the best fitted $\mathrm{mDW}$ algorithm we obtained the histogram of this quantity shown in fig 5, where the mean value of this ad-hoc defined 'sphericity' is 1.3 for C-jets and 1.6 for A-jets. These mean values are different and are bigger for A-jets due to the fact that some A-jets would be superposed interaction events, because the observation site is at $540 \mathrm{~g} / \mathrm{cm}^{2}$ level, that is the emulsion chamber is under 6 Nuclear Interaction lengths of Air. Events with 'sphericity' greater than 2 must be more carefully analysed because these values do not have a clear physical meaning.

\section{Sphericity s}

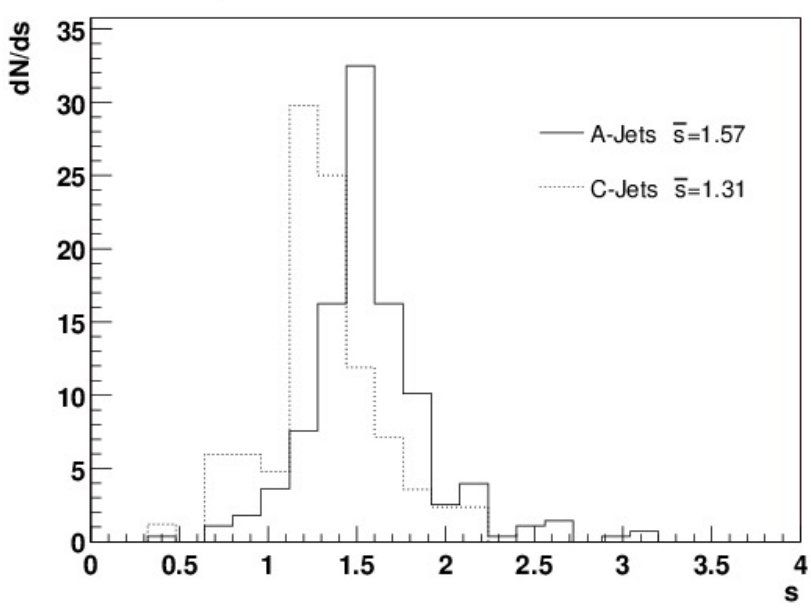

Figure 5. Distribution of 'sphericity'.

In fact, with the general expression above, it is also possible to calculate moments of order higher than 3 . In principle, the addition of these larger order moments to the algorithm mDW could smooth this curve. The difficulty comes from the normalization factor of these moments, for instance the $4^{\text {th }}$ moment has a $\log \left(1+\Gamma^{2} \theta^{2}\right)$ factor that does not converge at $\Gamma \theta \rightarrow \infty$.

\section{Analysis results}

C-jets analysis $[11,12]$ showed the correlation between $\gamma$ mean transverse momenta and rapidity density. This was also observed in the UA1/Cern experiment and we quote: 'This effect was not completely unexpected' written in a publication, Events of Very High Energy Density [13].

Analysis of A-jets shows the same correlation. Figure 4 shows this because the events with higher transverse momenta shift to the right.

The atmosphere above the chamber corresponds to 6 Nuclear Interaction length of air, so it is possible to observe A-jets with more than one interaction, whereas for $\mathrm{C}$-jets the probability to have more than one interaction is much less, taking into account that the Carbon target thickness is only around $23 \mathrm{~cm}$.

Assuming that the most energetic shower of Centauro $\mathrm{V}\left(\mathrm{n}^{\circ} 62=54.86 \mathrm{TeV}\right.$, and the second $\mathrm{n}^{\circ} 27=27.23$ $\mathrm{TeV}$ ) is the surviving particle of an interaction and that the tertiary produced particles are from normal multiple pion production, the characteristics of the interaction are [14]: Energy of primary particle $E_{0}=1,061 \mathrm{TeV}$, Inelasticity of collision $\mathrm{K}=0.81$, Mean inelasticity of $\gamma$-ray $<$ $k_{\gamma}>=0.27$, Hadron induced showers energy/Total energy $Q_{h}^{\prime}=0.90 \approx Q_{h}$, Rapidity density $N_{h} / \Delta Y=(8.56-9.89)$, Mean energy of secondary hadrons $\left\langle E_{h}\right\rangle=(21.5 \pm 4)$ $\mathrm{TeV}$, Mean transverse momentum $\left\langle P_{T_{h}}>=(1.2 \pm 0.2)\right.$ $\mathrm{GeV} / \mathrm{c}$. Without the surviving particle assumption, the values are: $E_{0}=873 \mathrm{TeV}, \mathrm{K}=1.0,<k_{\gamma}>=1 / 3$, $Q_{h}^{\prime}=0.90, N_{h} / \Delta Y=(8.32-9.34),<E_{h}>=(21 \pm 3.5) \mathrm{TeV}$, $\left.<P_{T_{h}}\right\rangle=(1.0 \pm 0.16) \mathrm{GeV} / \mathrm{c}$, results from identification of 25 and $37 \gamma$ and hadron induced showers, respectively. 


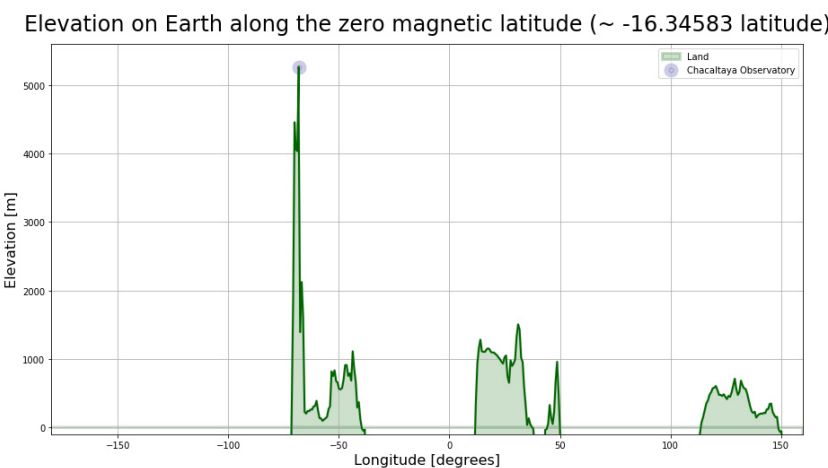

Figure 6. Elevation along the zero magnetic latitude.

Using another simulation for energy determination with $\chi^{2}>3.16$ best fitting results for 22 and 40 for $\gamma$ and hadron induced showers, respectively, and under the surviving particle assumption, the figures are: Energy of primary particle $E_{0}=1,047 \mathrm{TeV}$, Inelasticity of collision $\mathrm{K}=0.80$, Mean inelasticity of $\gamma$-ray $\left\langle k_{\gamma}>=0.27\right.$, Hadronic induced showers energy/Total energy $Q_{h}^{\prime}=0.89$ $\approx Q_{h}$, Rapidity density $N_{h} / \Delta Y=(10.25-13.19)$, Mean energy of secondary hadrons $\left\langle E_{h}\right\rangle=(19 \pm 3) \mathrm{TeV}$, Mean transverse momentum $<P_{T_{h}}>=(1.0 \pm 0.2) \mathrm{GeV} / \mathrm{c}$.

That is, we get almost similar figures independently of simulation and the mean transverse momentum for this Centauro event also does not depend on the quantity of identified hadrons.

Figure 6 shows that Chacaltaya and its surroundings are unique high altitude places on the Earth's surface, pointing to the Center of the Galaxy. Due to this fact, we can imagine that this would be the explanation for the quantity of halo containing events and that the halos are created by high energy $\gamma$ 's.

Table 1 shows that the fractional energy spectra for both $\gamma$ 's and hadron induced showers of 3 halo type events has similar behaviour as 5 Centauros events. The slopes for $\gamma$ and hadron events are different. Moreover the slope of $\gamma$ 's is steeper than for hadron showers, except for Centauro III. Previous results of the analysis of Centauro V [14] and the similarity shows that not only its relationships, but also that Centauros are not exotic events.

Remarkable A-jets are listed in Table 2. There is a subset of 18 events with $\Sigma E_{\gamma}>1,000 \mathrm{TeV}$. This subset is composed of 13 events with halo, therefore events with superposed interactions, plus 5 without a halo. Another subset of 13 events with $\Sigma E_{\gamma}>500 \mathrm{TeV}$ is shown as well as a further subset of 5 events called Centauro type events that have $\Sigma E_{\gamma}>200 \mathrm{TeV}$. There are many more higher energy events than in Table 1, therefore there are remaining events where we may look if the crossing behaviour of spectra would be a common signature in the $\Sigma E_{\gamma}>200$ $\mathrm{TeV}$ region. These 36 events were observed in 13 'runs', in a total exposure of $2,3 \times 10^{10} \mathrm{~m}^{2} . s$, under $\Omega=2 \pi / 8 \mathrm{sr}$ (the effective solid angle at Chacaltaya level).

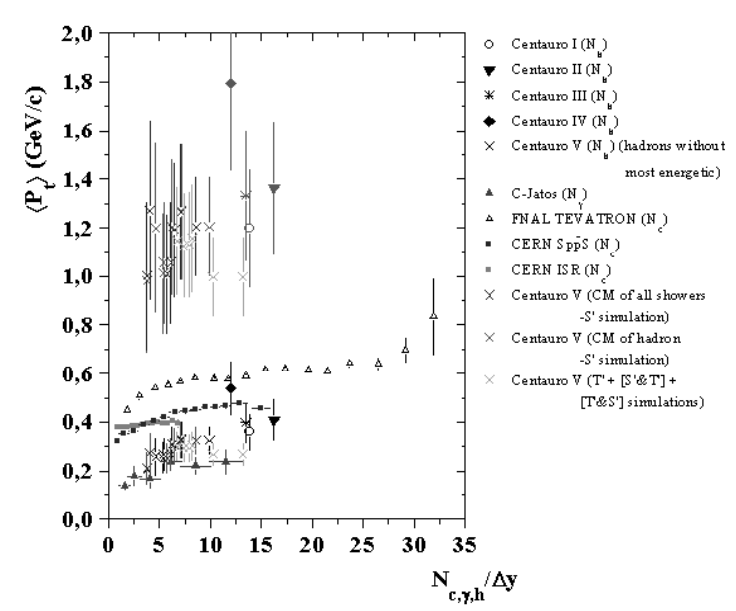

Figure 7. Mean $P_{T}$ - rapidity density correlation.

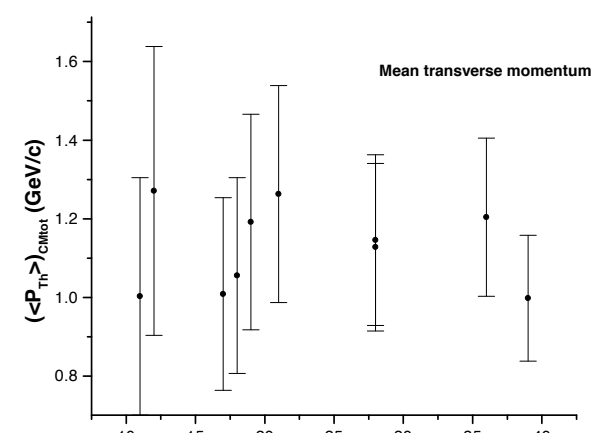

Figure 8. Mean transverse momentum of hadrons, estimated from the center of mass of all showers.

\section{Conclusions and discussions}

1) The publication [13] compared B-J Collaboration and SppS/CERN data. Transforming the $\gamma$ data of the B-J Collaboration to $\pi^{0}$, we got the correlation of the Mean Transverse Moment and Rapidity density shown in figure 7. In the same figure other data of cosmic ray and accelerator experiments is shown, showing the consistency.

2) The B-J Collaboration separated the events into two categories $\Sigma P_{T \gamma}<2.5 \mathrm{GeV} / \mathrm{c}$ and $\Sigma P_{T \gamma}>2.5 \mathrm{GeV} / \mathrm{c}$, but now it is better to separate events using the mean value of the transverse momenta. Then a signal of $\eta$-meson production was observed $[8,9]$ in the second group, corresponding to the plateau of figure 7 but not in the first group $\left(<P_{T \pi^{0}}>\right.$ less than $0.4 \mathrm{GeV} / \mathrm{c}$ ). A signal of $\eta$-meson production was observed in A-jets with a total energy of $28 \mathrm{TeV}$ and one $3 \pi^{0}$-meson decay event with an energy of $16 \mathrm{TeV}$ only in its $3 \pi^{0}$-meson. At the $20^{\text {th }}$ ISVHECRI-Nagoya, during the presentation "Recent results from LHCf/RHICf" by Takashi Sako, we were informed that the signal of $\eta$ meson production was observed in their experiments at 13 $\mathrm{TeV}$, but not at $7 \mathrm{TeV}$.

3) Similar values of energy distribution slopes, listed in Table 1 , possibly allows us to consider Centauros as normal events, not exotic ones. The only difference we observed is 
the mean transverse momenta of the order of $0.80 \mathrm{GeV} / \mathrm{c}$. In figure 7 data of Centauro $\mathrm{V}$ was inserted with many scenarios of hadron identification, plotted in figure 8 . It is seen that the mean transverse momentum of hadrons is almost constant, irrespective of the hadron identification procedure.

4) Maybe the 'selective' $\eta$-meson production supports Thermodynamical Models of Multiple Particle Production. At the time of ISR/CERN experiments a possibility was put forward supposing that the mean transverse momenta is in the order of the pion mass. An interesting paper, Thermodynamics of Strong Interactions at High Energy and its Consequences for Astrophysics [15], approaches this possibility.

5) Besides the previous comment, we may guess about halo containing events. The 13 events registered in 6 'runs', in $1,4 \times 10^{10} \mathrm{~m}^{2} . \mathrm{s}$ exposure of photosensitive material may be explained by the fact that Chacaltaya is on the geomagnetic equator. Moreover they are superposed interactions and the halo part is composed of electromagnetic particles yield at higher altitudes, maybe by $\gamma$.

\section{Acknowledgments}

The B-J Collaboration is indebted to various pioneers persons. We will name them in alphabetical order: Z.Ando, Y.Fujimoto, S.Hasegawa, M.Koshiba, C.M.G.Lattes, Y.Motoyama, J.Nishimura, S.Sakata, M.Taketani, S.Tomonaga, A.Tsukamoto, K.Wakisaka, S.Watanabe, H.Yukawa. Some stories, concerning the begining of the B-J Collaboration were these farmers, intellectuals and professors, are presented in Appendix B.

We have pleasure in acknowledging our indebtedness to the financial support from CBPF, CNEN, CNPq, FAEPUNICAMP, FAPESP, FINEP, UFF in Brazil and Aoyama Gakuin University, University of Tokyo, Waseda University, Ministry of Education in Japan. We are also grateful to IIF-UMSA in Bolivia, host of the experiments, for help on many occasions. The authors wish to acknowledge all members of the B-J Collaboration for the free use of the data. SLCB and AM wish to acknowledge the funding agency FAPESP for support (contracts no. 01/04150-0 and 04/03105-9, respectively).

\section{Appendix A}

Below, we list 6 moments obtained from a general expression in the text. For the purpose of this paper we used the first 4 moments, because the $5^{\text {th }}$ moment does not converge, in spite of the $6^{\text {th }}$ having a limit for $\Gamma \theta \rightarrow \infty$.

$$
\begin{gathered}
\Sigma E_{i}\left(\Gamma \theta_{i}\right)^{0} \simeq M \Gamma\left[1-\frac{1}{\left(1+\Gamma^{2} \theta^{2}\right)^{2}}\right] \\
\Sigma E_{i}\left(\Gamma \theta_{i}\right)^{1} \simeq M \Gamma\left[\frac{\arctan \Gamma \theta}{2}-\frac{\left(\Gamma \theta-\Gamma^{3} \theta^{3}\right)}{2\left(1+\Gamma^{2} \theta^{2}\right)^{2}}\right] \\
\Sigma E_{i}\left(\Gamma \theta_{i}\right)^{2} \simeq M \Gamma\left[\frac{\Gamma^{2} \theta^{2}}{1+\Gamma^{2} \theta^{2}}\right]^{2}
\end{gathered}
$$

$$
\begin{gathered}
\Sigma E_{i}\left(\Gamma \theta_{i}\right)^{3} \simeq M \Gamma\left[\frac{3 \arctan \Gamma \theta}{2}-\frac{3 \Gamma \theta+5 \Gamma^{3} \theta^{3}}{2\left(1+\Gamma^{2} \theta^{2}\right)^{2}}\right] \\
\Sigma E_{i}\left(\Gamma \theta_{i}\right)^{4} \simeq M \Gamma\left[2 \log \left(1+\Gamma^{2} \theta^{2}\right)^{2}-\frac{3 \Gamma^{4} \theta^{4}+2 \Gamma^{2} \theta^{2}}{\left(1+\Gamma^{2} \theta^{2}\right)^{2}}\right] \\
\Sigma E_{i}\left(\Gamma \theta_{i}\right)^{5} \simeq M \Gamma\left[\frac{3 \Gamma^{4} \theta^{4}-4 \Gamma^{3} \theta^{3}+18 \Gamma^{2} \theta^{2}+4 \Gamma \Theta+3}{\left(1+\Gamma^{2} \theta^{2}\right)^{2}}\right] \\
\times\left[\frac{\Gamma \Theta}{2}-\arctan \Gamma \theta\right]
\end{gathered}
$$

\section{Appendix B}

The proposal to start a cooperation between Brasil and Japan was sent by Hideki Yukawa after his visit in 1958, attending an invitation for the $50^{\text {th }}$ anniversary of Japanese Immigration. Before this visit, Brazilian Physicists inspired by the 1949 Nobel Prize of Physics to Hideki Yukawa, started a movement to invite his visit to Brasil. At the same time it was considered that his visit would be interesting to stop the bloodshed fighting between the so-called Loser and Winner Gangs (Make-Gumi and Kachi-Gumi) inside the Japanese immigrants community. Some immigrants regularly meet on Saturdays, to constitute the Saturday's Society (Doyo-Kai) where Yoshinori Motoyama suggested the name of Hideki Yukawa. To cover visit expenses they collected in the 1950s, $10^{6}$ Japanese Yens, that corresponds to 60,000-100,000 of today's American Dollars, mostly from farmers. Zempati Ando, living in a small farm at a town named Mizuho, cut eucalyptus trees and all the money was donated to this society. Mituo Taketani and Sin-Itiro Tomonaga was charged to receive this support sent through a Japanese newspaper Mainichi Shimbun, because Hideki Yukawa was at Columbia University from 1949 till 1953 and so unable to attend the invitation. This money was donated through a letter, dated $17^{\text {th }}$ August 1952 and signed by Ayami Tsukamoto and Shigueo Watanabe. In this letter many contributors are listed; among them we found the name of Katsunori Wakisaka, a close friend of B-J Collaboration. It was welcome and was used to promote the first international conference after the war and also to help experimental group of Cosmic Rays. Grateful words about this support is found in the Supplement of the Progress of Theoretical Physics, number 1(1955) in an editorial introduction by Sin-Itiro Tomonaga. Mituo Taketani expressed his gratefulness accepting a 1958 invitation to act as a scientific director and to improve activities at the Theoretical Physics Institute, São Paulo city. In 1958 Mituo Taketani and Hideki Yukawa visited Mizuho, where Zenpati Ando was cultivating eucalyptus trees. One of us (EHS) was told by Mituo Taketani about Zempati Ando, calling him Simpático Ando. Simpático is a Portuguese word, its pronunciation sounds Zempati and means sympathetical person.

These stories are not scientific ones, but it is very precious to Japanese Immigrants and descendants, stories preceding start of B-J Collaboration. The only document for 
the scientific cooperation on Cosmic Ray experiments between both countries is the proposal mentioned beforehand and it ran with goodwill of both sides. One of the authors, (EHS) would like to say that we are proud to register these stories and declare that he is very honored with reception of Mituo Taketani at all occasions of visit to Japan.

\section{References}

[1] G.Wataghin, Comptes Rendus des Séances de l'Académie des Sciences297,(juin1938),358-360

[2] G.Photzer, Z.Physik102,(1936),(23-58)

[3] J.J.Lord, J.Fainberg and M.Scheim, Phys.Rev.80,970(1950)

[4] Kusumoto C., S.Miyake, K.Suga and Y.Watase, Phys.Rev.90,998(1953)

[5] I.C.E.F. COLLABORATION, Nuovo Cimento, Supp.I,N.4,1039-1090(1963)
[6] J.Nishimura, Soryushiron Kenkyu (mimeographed circular in Japanese)12,24(1956)

[7] M.Luksys, A.Ohsawa, E.H.Shibuya, Nucl.Inst.\& Meth. B82 (1983),549-556

[8] Shibuya,E.H., Proc. XVIII ${ }^{\text {th }}$ ICRC,vol.11,(1983),97

[9] Sérgio Luiz Carmelo Barroso, Busca de mésons $\eta$ (Master thesis approved by IFGW/UNICAMP(1996)

[10] N.M.Duller and W.D.Walker, Phys.Rev.93,No.1,(1954),215

[11] Brasil-Japan Collaboration of Chacaltaya Emulsion Chamber Experiment, Prog.Theo.Phys.Sup76, 1(1983)

[12] Hiroshi Kumano, Prog.Theo.Phys.Sup76, 51(1983)

[13] G.Pancheri and C.Rubbia, Nucl.Phys.A418, 117(1984)

[14] S.L.C.Barroso, P.C.Beggio, A.O.de Carvalho, J.A.Chinellato, A.Mariano, R.de Oliveira, E.H.Shibuya, Nuclear Physics B (Proc.Suppl.)175+176 (2008) 131-136

[15] R.Hagedorn, Astron. \& Astrophys.5, 184-205(1970) 


\begin{tabular}{|c||c||c|c|}
\hline \multicolumn{1}{|c||}{ Run number' } & nickname & Slope of $\gamma$ 's & Slope of hadrons \\
\hline C14-126 & Andromeda & $-1.74 \pm 0.08$ & $-0.89 \pm 0.04$ \\
\hline C15s175i137 & Ursa Major & $-1.71 \pm 0.07$ & $-0.9 \pm 0.1$ \\
\hline C19s152i083 & Mini-Andromeda III & $-2.2 \pm 0.2$ & $-1.26 \pm 0.06$ \\
\hline C21s(087+088+100+101)i(064+074+075) & Centauro VII & $-1.69 \pm 0.09$ & $-1.20 \pm 0.04$ \\
\hline C15s055i012 & Centauro I & - & $-0.59 \pm 0.03$ \\
\hline C16s107i090 & Centauro V & $-2.9 \pm 0.2$ & $-1.17 \pm 0.06$ \\
\hline C17s070i059 17 s097i070 & Centauro II & $-1.3 \pm 0.4$ & $-0.87 \pm 0.04$ \\
\hline C17s215i137 & Centauro III & $-0.66 \pm 0.04$ & $-0.78 \pm 0.04$ \\
\hline & Centauro IV & $-0.88 \pm 0.04$ & $-0.72 \pm 0.04$ \\
\hline
\end{tabular}

Table 1. Slopes of fractionally energy spectra, $\mathrm{E} / \Sigma E$

\begin{tabular}{|c|c|c|c|}
\hline \multirow[t]{2}{*}{ 'Run number' } & \multirow[t]{2}{*}{ nickname } & \multicolumn{2}{|c|}{$\Sigma E_{\gamma}(\mathrm{TeV})$} \\
\hline & & showers & halo \\
\hline C14-126 & Andromeda & $6,144.24$ & 21,000 \\
\hline C14-181 & Magelan & $1,876.00$ & 980. \\
\hline C15s175i137 & Ursa Major & $2,394.85$ & 980. \\
\hline C16s093 & & $2,157.00$ & 290. \\
\hline $\mathrm{C} 17 \mathrm{~s} 015$ & & $1,479.00$ & - \\
\hline $\mathrm{C} 18 \mathrm{~s} 086$ & & $>1,132.00$ & $>2,300$ \\
\hline C18s154i133 & & $1,414.03$ & - \\
\hline C18sP06i074 & & $1,274.40$ & 1,000 \\
\hline $\mathrm{C} 18 \mathrm{~A}$ & Mini-Andromeda II & 889.04 & 1,300 \\
\hline $\mathrm{C} 18 \mathrm{~A}$ & Mini-Andromeda I & $1,334.94$ & 3,200 \\
\hline C19s174i134 & & $1,163.98$ & 150. \\
\hline C19s213i155 & & $1,088.07$ & - \\
\hline C19s152i083 & Mini-Andromeda III & $3,703.34$ & 5,100 \\
\hline C19A032 & & $1,138.00$ & 286. \\
\hline C19A127 & & 222.00 & 1,380 \\
\hline C20s107i090 & Centauro VI & $1,249.40$ & - \\
\hline $\mathrm{C} 21 \mathrm{~s}(087+088+100+101) \mathrm{i}(064+074+075)$ & Centauro VII & $5,237.55$ & 19,800 \\
\hline C22s113i084 & & $1,351.70$ & - \\
\hline C18s021i003 & & $\overline{550.00}$ & \\
\hline C18s057i035 & & 519.30 & \\
\hline C18s101-F1 & & 572.10 & \\
\hline C18s131i115 & & 916.05 & \\
\hline C18s185i149 & & 843.83 & \\
\hline C19s179i126 & & 754.74 & \\
\hline C19s011i021 & & 785.45 & \\
\hline C19s215i159 & & 554.80 & \\
\hline C20s018i:out & & 908.50 & \\
\hline C22s147i106 & & 586.70 & \\
\hline C22s148i102 & & 490.30 & \\
\hline C22s149i094 & & 594.70 & \\
\hline C22s178i139 & & 814.78 & \\
\hline 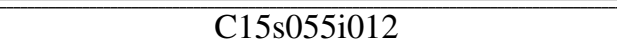 & Centauro I & 230.53 & \\
\hline C16s107i090 & Centauro V & $291.50+54.86$ & \\
\hline C17s070i059 & Centauro II & 203.37 & \\
\hline C17s097i070 & Centauro III & 269.82 & \\
\hline C17s215i137 & Centauro IV & 441.44+halo? & \\
\hline
\end{tabular}

Table 2. Relevant A-jets, 18 with $\Sigma E_{\gamma}>1,000 \mathrm{TeV}, 13$ with $\Sigma E_{\gamma}>500 \mathrm{TeV}$ and 5 Centauro events with $\Sigma E_{\gamma}>200 \mathrm{TeV}$ 